\title{
Hand-sewn versus stapled anastomoses for esophagectomy: We will probably never know which is better
}

\author{
Tommi Järvinen, MD, PhD, ${ }^{\mathrm{a}, \mathrm{b}}$ Jonathan Cools-Lartigue, $\mathrm{MD},{ }^{\mathrm{c}}$ Eric Robinson, MD, BS, \\ Jari Räsänen, $\mathrm{MD}, \mathrm{PhD},{ }^{\mathrm{a}, \mathrm{b}}$ and Ilkka Ilonen, $\mathrm{MD}, \mathrm{PhD}^{\mathrm{a}, \mathrm{b}}$
}

\begin{abstract}
Objective: Esophagectomy remains the mainstay of treatment for nonmetastatic esophageal cancer. The optimal technique for anastomosis after esophagectomy remains unknown. The purpose of this systematic meta-analysis is to combine the available high-quality evidence to provide esophageal surgeons with an evidence base for their decision making.
\end{abstract}

Methods: A systematic search of multiple databases was conducted to find randomized controlled trials of esophageal anastomotic techniques. A meta-analysis of the pooled data was conducted.

Results: A total of 19 studies with 2123 patients were included in the meta-analysis. The pooled analysis revealed a 102\% higher incidence of anastomotic leak after hand-sewn anastomosis compared with stapled anastomosis (odds ratio [OR], 2.02; 95\% confidence interval [Cl], 1.48-2.75). Anastomotic stricture rate was also $31 \%$ higher with hand-sewn anastomosis (OR, 1.31; $95 \% \mathrm{Cl}, 1.00-1.7)$. Thirty-day mortality did not show statistical difference favoring one anastomosis technique to another (OR, 0.68; 95\% Cl, 0.45-1.04). None of anastomotic leak rate, anastomotic stricture rate, or 30-day overall survival differed between anastomotic techniques in studies with only thoracic anastomoses. In cervical position hand-sewn anastomosis was associated with higher rate of anastomotic leak (OR, 2.02; 95\% $\mathrm{Cl}, 1.33-3.05)$ and stricture (OR, $1.77 ; 95 \% \mathrm{Cl}, 1.15-2.72)$, but no difference in 30day mortality.

Conclusions: This meta-analysis showed a signal of higher rate of leak and stricture in hand-sewn anastomoses, but sensitivity analyses did not show a consistent outcome, so these results should be interpreted with caution. (JTCVS Open 2021;7:338-52)

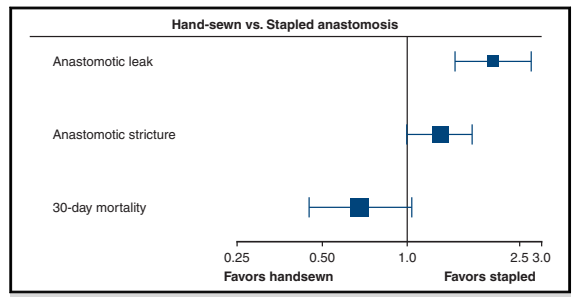

Forest plot for rate of anastomotic leakage between hand-sewn and stapled anastomosis.

\section{CENTRAL MESSAGE}

A higher rate of leakage and

stricture with hand-sewn anas-

tomotic technique was found in

this meta-analysis. The studies

were heterogeneous, and with

variance in results of the sensi-

tivity analyses.

\section{PERSPECTIVE}

The rate of anastomotic leakage after esophagectomy remains significant, and there is no consensus on the optimal anastomotic technique. This meta-analysis provides a summary of the current evidence, highlighting the need for more quality studies, especially in minimally invasive settings.

See Commentaries on pages 353 and 355 .
From the ${ }^{\mathrm{a} D e p a r t m e n t}$ of General Thoracic and Esophageal Surgery, Helsinki University Hospital, Helsinki, Finland; ${ }^{b}$ Department of Surgery, Clinicum, University of Helsinki, Helsinki, Finland; ${ }^{\circ}$ Department of Thoracic and Upper GI surgery, Montreal General Hospital, Montreal, Québec, Canada; and ${ }^{\mathrm{d}}$ Department of Thoracic Surgery, Icahn School of Medicine at Mount Sinai, New York, NY.

Received for publication Nov 24, 2020; accepted for publication July 21, 2021; available ahead of print Aug 13, 2021

Address for reprints: Tommi Järvinen, MD, PhD, Department of General Thoracic and Esophageal Surgery, Heart and Lung Center, Helsinki University Hospital, HUS, PO Box 340, FIN-00029 Helsinki, Finland (E-mail: tommi.jarvinen@ helsinki.fi).

2666-2736

Copyright (C) 2021 The Author(s). Published by Elsevier Inc. on behalf of The American Association for Thoracic Surgery. This is an open access article under the CC BY-NC-ND license (http://creativecommons.org/licenses/by-nc-nd/4.0/).

https://doi.org/10.1016/j.xjon.2021.07.021 $\checkmark$ Video clip is available online.

Esophagectomy_-Ivor Lewis, McKeown, or transhiatal-is the established treatment for locoregional esophageal cancer. After esophageal resection, the reconstruction is generally performed by gastric pull-up and either intrathoracic or cervical esophagogastric anastomosis. ${ }^{1}$ The standard approach has historically been open esophagectomy, but during the past few decades minimally invasive esophagectomy has gained popularity, with benefits over open esophagectomy in regard to overall and disease-specific survival, 


\section{Abbreviations and Acronyms \\ $\mathrm{AL}=$ anastomotic leak \\ OS = overall survival \\ $\mathrm{RCT}=$ randomized controlled trial}

pulmonary complications, quality of life, and hospital stay. $^{2-4}$ Anastomotic leak (AL) is a devastating complication with a relatively high incidence; a recent meta-analysis of randomized trials reported an incidence of $11.2 \%{ }^{5,6}$ The development and adoption of minimally invasive techniques raises the questions regarding optimal anastomotic technique because hand-sewn anastomosis is challenging by the thoracoscopic/laparoscopic approach. There is a paucity of high-quality evidence examining the effect of anastomotic technique on the development of AL, particularly in the minimally invasive setting.

The purpose of this meta-analysis was to determine whether contemporary evidence highlights superiority of 1 anastomotic technique (hand-sewn vs mechanical stapler) over another with respect to the development of AL, anastomotic stricture, and overall survival (OS).

\section{METHODS}

\section{Design}

This is a systematic literature review and meta-analysis that followed a predetermined study protocol according to Preferred Reporting Items for Systematic Reviews and Meta-Analyses guidelines for systematic reviews and meta-analyses.

\section{Literature Search Strategy}

Two independent investigators conducted a systematic literature search contemporary to June 2020 from multiple databases (Embase, Medline, and Cochrane library). The search was performed by combining medical subject headings and related free-text search terms with Boolean operators "AND" or "OR." The MeSH terms used were esophageal neoplasms and anastomosis, surgical. A full description of the search strategy is available in Table E1.

\section{Study Selection}

The inclusion criteria were as follows: randomized controlled study, study patients underwent esophagectomy, study compared different anastomotic techniques, and study reported anastomotic leak and/or stricture outcomes. Exclusion criteria were English translation of the manuscript not available.

\section{Data Extraction}

Titles and abstracts were scrutinized by the first author (T.J.) and duplicates were identified simultaneously. Full texts of potential studies were analyzed by 2 authors (T.J. and I.I.). Summary data were extracted from included studies. Extracted data included publication year, sample size, tumor location and histology, operative technique, anastomosis technique, follow-up, 30-day mortality, hospital mortality, AL, and stricture rate.

\section{Quality Assessment}

Quality of studies was assessed by 1 author (T.J.) using Cochrane Collaborations Risk of Bias Tool for randomized clinical trials, which is presented in Table E2.

\section{Informed Consent}

Because this was a meta-analysis that does not process individual patient data, no informed consent, as per Helsinki University Institutional Review Board guidelines, was needed.

\section{Outcome Measures and Statistical Analysis}

The primary outcome measures evaluated were odds ratios (OR) for AL rate and stricture. The secondary outcome measure was hazard ratio for 30day mortality. Subset analyses dichotomizing patients according to anastomotic location (cervical vs thoracic) was performed.

Meta-analysis of data was conducted using a random effects model due to high heterogeneity. Publication bias was assessed by funnel plots (plots of effect estimates against sample size) to detect outliers or asymmetry. Funnel plot asymmetry was analyzed visually and by Egger test for small-study effects and publication bias. The statistical significance for $\mathrm{Eg}$ ger test was set at $P<.10$, as originally described by Egger and colleagues. ${ }^{8}$ To maximize the number of patients included in the meta-analysis, studies that investigated subgroups within either only hand-sewn or only stapled anastomoses were included in the summary quantitative synthesis representing only a single group, either hand-sewn or stapled anastomosis, with the opposing group size being 0 . Forest plots; that is, graphical display of estimated ORs and $95 \%$ confidence intervals (CIs) and summary statistics were used to elucidate the results of the studies. Post hoc sensitivity analyses were performed without the inclusion of these single-group studies to investigate the robustness of this analysis. Sensitivity analyses were also performed with studies containing only thoracic anastomoses or only cervical anastomoses and also without the inclusion of studies predating 2000 to elucidate the possible differences of outcomes within these subgroups.

The $I^{2}$ test was used to evaluate statistical heterogeneity, also known as the outcome variability in excess of what would be expected due to measurement error alone of the included studies, with levels of heterogeneity defined as not important $\left(I^{2}, 0 \%-25 \%\right)$, moderate $\left(I^{2}, 25 \%-50 \%\right)$, substantial $\left(I^{2}, 50 \%-75 \%\right)$, or considerable $\left(I^{2}, 75 \%-100 \%\right)$. Statistical analysis was done with R version 2020 (R Foundation for Statistical Computing, Vienna, Austria).

\section{RESULTS}

The database search generated 3153 study titles, of which 19 studies met the inclusion criteria (Figure 1). The anastomotic approaches used were evenly distributed: 1160 $(50.3 \%)$ hand-sewn anastomoses and $1148(49.7 \%)$ mechanical stapler anastomoses. Eight of the 19 studies $(42.1 \%)$ included only cervical anastomoses, ${ }^{9-16} 6$ studies $(31.6 \%)$ included only thoracic anastomoses, ${ }^{17-22}$ and 5 studies $(26.3 \%)$ had both cervical and thoracic anastomoses. $^{23-27}$ Two of the studies were randomized trials of antireflux anastomotic techniques, of which $1 \mathrm{did}$ not report any of the prespecified outcomes between hand-sewn and stapled groups and the other had no adequate text in English available, so both were excluded from the summary statistics. ${ }^{20,21}$ Remaining studies included 2230 patients across 17 studies. Characteristics of the studies are presented in Table 1 .

The studies had somewhat differing definitions for AL, although all of the studies used routine radiographic imaging on postoperative day 3 through 10 . Stricture definitions differed somewhat between studies, the most common definitions being the inability to pass a small diameter (9$10 \mathrm{~mm}$ ) enteroscope past the anastomosis, a small diameter 


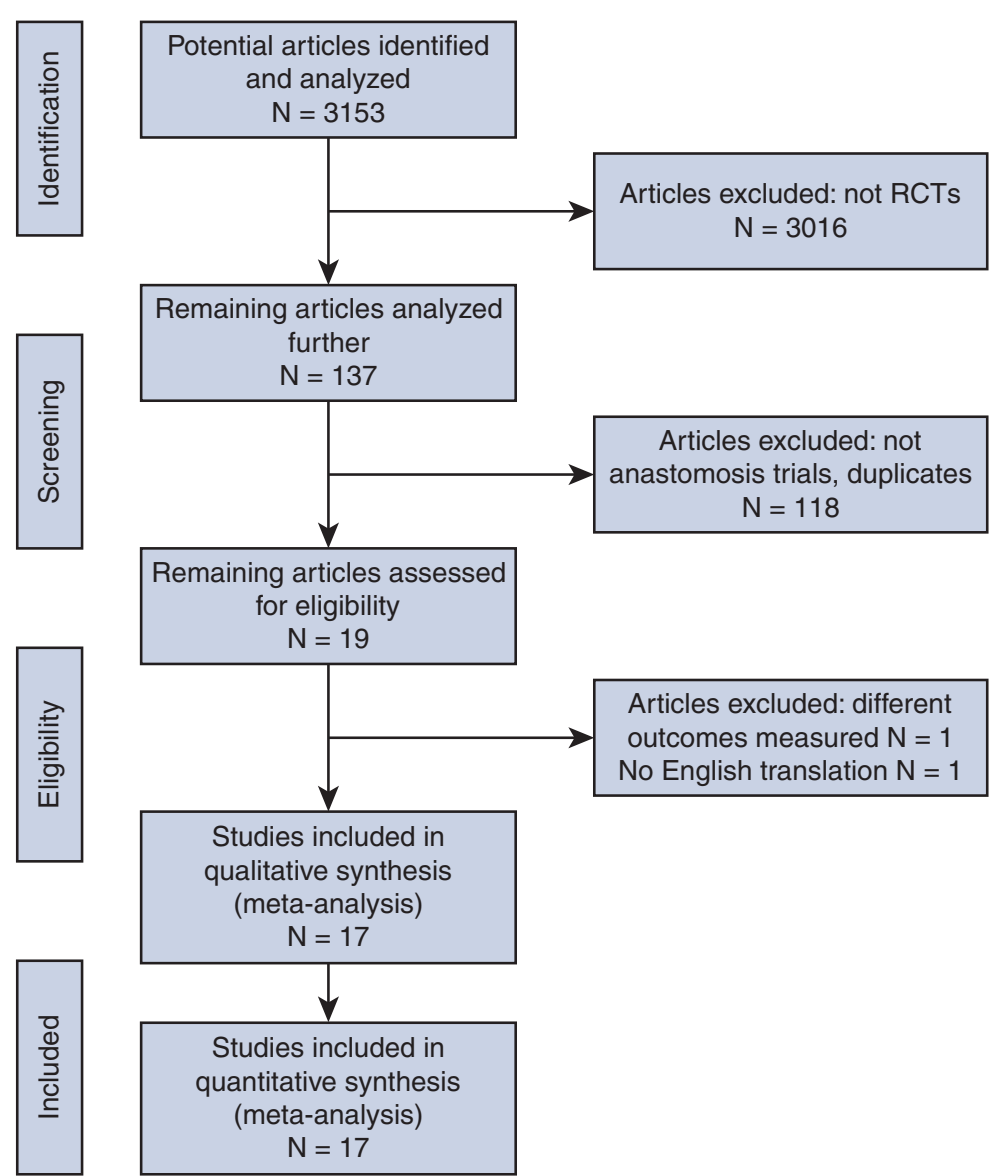

FIGURE 1. Flow chart of the literature search according to preferred reporting items for systematic reviews and meta-analyses statement. RCTs, Randomized controlled trials.

of the anastomosis $(<8-10 \mathrm{~mm})$ in imaging or the need for dilatation based on symptoms. The definitions of AL and anastomotic stricture and related methods of diagnosis of each study are presented in Table 2 .

All 17 studies reported AL rates. The pooled analysis revealed a $102 \%$ higher incidence of $\mathrm{AL}$ after hand-sewn anastomosis compared with stapled anastomosis (OR, 2.02; 95\% CI, 1.48-2.75). Figure 2 shows the associated forest plot. Statistical heterogeneity of the studies was moderate $(I 2,42 \% ; P=.08)$. Funnel plot showed symmetry visually and statistically (Egger test, 0.87). The funnel plot is displayed in Figure 3, A.

Anastomotic stricture rates were reported by all but 1 study. Hand-sewn anastomosis group had a $31 \%$ increased incidence of anastomotic stricture; however, the statistical significance was borderline significant (OR, 1.31; 95\% CI, 1.00-1.7). The forest plot is presented in Figure 4. Statistical heterogeneity was substantial $(I 2,58 \% ; P=.006)$. Visual and statistical symmetry was confirmed by funnel plot (Egger test, 0.20). The funnel plot is presented in Figure 3, $B$.

Thirty-day mortality was reported only by $9(52.9 \%)$ studies. Thirty-day mortality did not show statistical difference favoring 1 anastomosis technique over another (OR, $0.68 ; 95 \%$ CI, 0.45-1.04). Figure 5 displays the related forest plot. Statistical heterogeneity of these studies was not important $(I 2,22 \% ; P=.35)$. Funnel plot was symmetrical visually and by statistical analysis (Egger test, 0.39). Figure $3, C$, presents the funnel plot.

\section{Sensitivity Analyses}

Figure 6 shows the summary statistics of the sensitivity analyses. Without single-group studies, the leak rate and stricture rate were not statistically different, but the 30day mortality rate favored the hand-sewn anastomosis (OR, 0.52; 95\% CI, 0.33-0.84). When studies predating 2000 were excluded, the results did not change from the original analysis: leak rate favored stapled anastomosis 
TABLE 1. Characteristics of included randomized controlled trials

\begin{tabular}{|c|c|c|c|c|c|c|c|c|c|}
\hline \multirow[b]{2}{*}{ Study } & \multicolumn{6}{|c|}{ Characteristic } & \multicolumn{3}{|c|}{$\mathbf{N}$} \\
\hline & Country & $\begin{array}{c}\text { Location } \\
\text { of the } \\
\text { anastomosis }\end{array}$ & $\begin{array}{c}\text { Surgical } \\
\text { approaches }\end{array}$ & Group A & Group B & Group C & $\begin{array}{c}\text { Group } \\
\text { A }\end{array}$ & $\begin{array}{c}\text { Group } \\
\text { B }\end{array}$ & $\begin{array}{l}\text { Group } \\
\text { C }\end{array}$ \\
\hline $\begin{array}{c}\text { Ribet et al, } \\
1992^{23}\end{array}$ & France & Both & $\mathrm{IL}^{*}$ and $\mathrm{McK}^{\dagger}$ & $\begin{array}{l}\text { Hand sewn, } \\
\text { Cervical }\end{array}$ & Hand sewn, Thoracic & & 30 & 30 & \\
\hline $\begin{array}{c}\text { Zieren et al, } \\
1993^{\circ}\end{array}$ & Germany & Cervical & $\begin{array}{r}\mathrm{IL}^{*}, \mathrm{McK}_{\dagger} \\
\text { and } \mathrm{TH} \ddagger\end{array}$ & $\begin{array}{l}\text { 1-layer } \\
\text { hand sewn }\end{array}$ & 2-layer hand sewn & & 54 & 52 & \\
\hline $\begin{array}{c}\text { Bardini et al, } \\
1994^{10}\end{array}$ & Italy & Cervical & $\mathrm{TH}+$ & $\begin{array}{l}\text { Hand sewn, } \\
\text { continuous }\end{array}$ & Hand sewn, interrupted & & 21 & 21 & \\
\hline $\begin{array}{l}\text { Valverde et al, } \\
1996^{24}\end{array}$ & France & Both & $\mathrm{IL}^{*}, \mathrm{McK}_{\dagger} \mathrm{TH}_{\ddagger}^{\dagger}$ & Hand sewn & Stapled & & 74 & 78 & \\
\hline $\begin{array}{l}\text { Law et al, } \\
1997^{17}\end{array}$ & China & Thoracic & $\mathrm{IL}^{*}$ & Hand sewn & Circular stapled & & 61 & 61 & \\
\hline $\begin{array}{c}\text { Laterza et al, } \\
1999^{11}\end{array}$ & Italy & Cervical & $\mathrm{McK}_{\dagger}^{\dagger}$ & Hand sewn & Stapled & & 21 & 20 & \\
\hline $\begin{array}{l}\text { Walther et al, } \\
2003^{25}\end{array}$ & Sweden & Both & $\mathrm{IL}^{*}$ and $\mathrm{McK}^{\dagger}$ & $\begin{array}{c}\text { Hand sewn, } \\
\text { Cervical }\end{array}$ & $\begin{array}{l}\text { Circular stapled, } \\
\text { Thoracic }\end{array}$ & & 41 & 42 & \\
\hline $\begin{array}{l}\text { Hsu et al, } \\
2004^{15}\end{array}$ & Taiwan & Cervical & $\mathrm{McK}_{\dagger}$ and $\mathrm{TH}_{\dagger}^{\dagger}$ & Hand sewn & Circular stapled & & 32 & 31 & \\
\hline $\begin{array}{l}\text { Okuyama } \\
\text { et al, } 2007^{26}\end{array}$ & Japan & Both & $\mathrm{IL}^{*}$ and $\mathrm{McK}_{\dagger}^{\dagger}$ & $\begin{array}{l}\text { Hand sewn, } \\
\text { Cervical }\end{array}$ & $\begin{array}{l}\text { Circular stapled, } \\
\text { Thoracic }\end{array}$ & & 18 & 14 & \\
\hline $\begin{array}{c}\text { Luechakiettisak } \\
\text { et al, } 2008^{18}\end{array}$ & Thailand & Thoracic & $\mathrm{IL}^{*}$ & Hand sewn & Circular stapled & & 59 & 58 & \\
\hline $\begin{array}{l}\text { Zhang et al, } \\
2010^{19}\end{array}$ & China & Thoracic & L Thoracotomy & Hand sewn & Circular stapled & & 244 & 272 & \\
\hline $\begin{array}{r}\text { Aly et al, } \\
2010^{20}\end{array}$ & Australia & Thoracic & $\mathrm{IL}^{*}$ & $\begin{array}{l}\text { Stapled with } \\
\text { fundoplication }\end{array}$ & $\begin{array}{l}\text { Stapled without } \\
\text { fundoplication }\end{array}$ & & 29 & 27 & \\
\hline $\begin{array}{l}\text { Ma et al, } \\
2010^{27}\end{array}$ & China & Both & N/A & Hand sewn & Stapled, side-to-side & $\begin{array}{l}\text { Stapled, } \\
\quad \text { circular }\end{array}$ & 52 & 45 & 47 \\
\hline $\begin{array}{l}\text { Nederlof } \\
\quad \text { et al, } 2011^{12}\end{array}$ & $\begin{array}{l}\text { The } \\
\text { Netherlands }\end{array}$ & Cervical & $\mathrm{IL}^{*} \& \mathrm{TH}+$ & $\begin{array}{l}\text { Hand sewn, } \\
\text { end to side }\end{array}$ & Hand sewn, end-to-end & & 64 & 64 & \\
\hline $\begin{array}{r}\text { Liu et al, } \\
2011^{21}\end{array}$ & China & Thoracic & N/A & $\begin{array}{l}\text { Stapled with } \\
\text { fundoplication }\end{array}$ & $\begin{array}{l}\text { Stapled without } \\
\text { fundoplication }\end{array}$ & & 35 & 35 & \\
\hline $\begin{array}{l}\text { Saluja et al, } \\
2012^{13}\end{array}$ & India & Cervical & $\mathrm{McK}_{\dagger} \dagger$ & Hand sewn & Side to side stapled & & 87 & 87 & \\
\hline $\begin{array}{c}\text { Cayi et al, } \\
2012^{14}\end{array}$ & China & Cervical & N/A & Hand sewn & Stapled & & 125 & 125 & \\
\hline $\begin{array}{l}\text { Wang et al, } \\
2013^{22}\end{array}$ & China & Thoracic & L Thoracotomy & Hand sewn & Circular stapled & $\begin{array}{l}\text { Semi- } \\
\text { mechanical }\end{array}$ & 52 & 47 & 45 \\
\hline $\begin{array}{l}\text { Hayata et al, } \\
2017^{16}\end{array}$ & Japan & Cervical & Hybrid \& $\mathrm{McK}_{\dagger} \dagger$ & $\begin{array}{l}\text { Ciruclar } \\
\text { stapled }\end{array}$ & Triangular linear stapled & & 49 & 51 & \\
\hline
\end{tabular}

IL, Ivor Lewis; $M c K$, McKeown; $T H$, transhiatal; N/A, not available. *Ivor Lewis esophagectomy. †McKeown esophagectomy. $\ddagger$ Transhiatal esophagectomy

(OR, 2.37; 95\% CI, 1.63-3.43), no difference in stricture rate or mortality.

When analyzing thoracic anastomoses only, including 4 studies, neither AL rate (OR, 1.74; 95\% CI, 0.95-3.17), anastomotic stricture rate (OR, 0.73; 95\% CI, 0.42-1.28) nor 30-day OS rate (OR, 0.48; 95\% CI, 0.15-1.57) differed between anastomotic techniques. ${ }^{17-19,22}$ Figure E1 shows the associated forest plot.

In cervical position, analysis of 8 studies showed that hand-sewn anastomosis was associated with higher rate of AL (OR, 2.02; 95\% CI, 1.33-3.05) and stricture (OR, 1.77 ; $95 \% \mathrm{CI}, 1.15-2.72$ ), but no difference in 30-day OS 
TABLE 2. Identification and definition of anastomotic leak and anastomotic stricture in the included studies

\begin{tabular}{|c|c|c|c|c|}
\hline Study & Anastomotic leak diagnosis & $\begin{array}{c}\text { Routine postoperative } \\
\text { anastomotic assessment }\end{array}$ & Stricture diagnosis & $\begin{array}{c}\text { Last } \\
\text { follow-up }\end{array}$ \\
\hline Ribet et al, $1992^{23}$ & Any radiographic evidence & $\begin{array}{l}\text { 7th day postoperative swallow } \\
\text { study* }\end{array}$ & N/A & N/A \\
\hline Zieren et al, $1993^{9}$ & Any radiographic evidence & $\begin{array}{l}\text { 7th day postoperative swallow } \\
\text { study }\end{array}$ & $\begin{array}{l}\text { - Any form of anastomotic } \\
\text { narrowing requiring endoscopic } \\
\text { dilatation or operative revision } \\
\text { - Inability to proceed into the gastric } \\
\text { tube with a } 9 \mathrm{~mm} \text { endoscope }\end{array}$ & $\begin{array}{l}\text { N/A (mean } \\
\text { follow-up } \\
\text { of } 44 \mathrm{wk} \text { ) }\end{array}$ \\
\hline Bardini et al, $1994^{10}$ & Any radiographic evidence & $\begin{array}{l}\text { 10th day postoperative } \\
\text { swallow study }\end{array}$ & $\begin{array}{l}\text { - Radiographic anastomotic } \\
\text { diameter }<1 \mathrm{~cm} \\
\text { - Any dysphagia }\end{array}$ & $3 \mathrm{mo}$ \\
\hline Valverde et al, $1996^{24}$ & $\begin{array}{l}\text { Drain output of intestinal fluids } \\
\text { or orally ingested methylene } \\
\text { blue } \\
\text { - Any radiographic evidence } \\
\text { - Repeat operation or autopsy }\end{array}$ & $\begin{array}{l}\text { 3-8 postoperative swallow } \\
\text { study and methylene blue }\end{array}$ & $\begin{array}{l}\text { Any form of anastomotic } \\
\text { narrowing requiring endoscopic } \\
\text { dilatation or operative revision }\end{array}$ & $3 \mathrm{mo}$ \\
\hline Law et al, $1997^{17}$ & $\begin{array}{l}\text { - Any radiographic evidence } \\
\text { - Any endoscopic evidence }\end{array}$ & $\begin{array}{l}\text { 7th day postoperative swallow } \\
\text { study and endoscopy }\end{array}$ & $\begin{array}{l}\text { - Inability to proceed into the gastric } \\
\text { tube with a } 10 \mathrm{~mm} \text { endoscope }\end{array}$ & $1 \mathrm{y}$ \\
\hline Laterza et al, $1999^{11}$ & $\begin{array}{l}\text { Drain output of intestinal fluids } \\
\text { or orally ingested methylene } \\
\text { blue } \\
\text { - Any radiographic evidence }\end{array}$ & $\begin{array}{l}\text { postoperative day } 9-10 \\
\text { swallow study and } \\
\text { methylene blue }\end{array}$ & $\begin{array}{l}\text { Any form of anastomotic } \\
\text { narrowing requiring endoscopic } \\
\text { dilatation or operative revision }\end{array}$ & At least $6 \mathrm{mo}$ \\
\hline Walther et al, $2003^{25}$ & $\begin{array}{l}\text { - Drain output of intestinal fluids } \\
\text { - Any radiographic evidence }\end{array}$ & $\begin{array}{l}\text { 5th day postoperative swallow } \\
\text { study }\end{array}$ & $\begin{array}{l}\text { - Inability to proceed into the gastric } \\
\text { tube with a } 9 \mathrm{~mm} \text { endoscope }\end{array}$ & $1 \mathrm{y}$ \\
\hline Hsu et al, $2004^{15}$ & $\begin{array}{l}\text { - Drain output of intestinal fluids } \\
\text { - Any radiographic evidence }\end{array}$ & $\begin{array}{l}\text { 7-10 d postoperative swallow } \\
\text { study }\end{array}$ & $\begin{array}{l}\text { - Inability to proceed into the gastric } \\
\text { tube with a } 10 \mathrm{~mm} \text { endoscope }\end{array}$ & $\begin{array}{l}\text { N/A (24 mo } \\
\text { follow-up } \\
\text { mean) }\end{array}$ \\
\hline Okuyama et al, $2007^{26}$ & Any radiographic evidence & $\begin{array}{l}\text { 9-10 d postoperative swallow } \\
\text { study }\end{array}$ & $\begin{array}{l}\text { Any form of anastomotic } \\
\text { narrowing requiring endoscopic } \\
\text { dilatation or operative revision }\end{array}$ & $6 \mathrm{mo}$ \\
\hline $\begin{array}{l}\text { Luechakiettisak } \\
\text { et al, } 2008^{18}\end{array}$ & Any radiographic evidence & $\begin{array}{l}\text { 7th day postoperative swallow } \\
\text { study }\end{array}$ & $\begin{array}{l}\text { - Inability to proceed into the gastric } \\
\text { tube with an endoscope }\end{array}$ & $3 \mathrm{mo}$ \\
\hline Zhang et al, $2010^{19}$ & $\begin{array}{l}\text { - Drain output of intestinal fluids } \\
\text { - Any radiographic evidence }\end{array}$ & $\begin{array}{l}\text { 5-10 d postoperative swallow } \\
\text { study }\end{array}$ & $\begin{array}{l}\text { - Inability to proceed into the gastric } \\
\text { tube with a } 10 \mathrm{~mm} \text { endoscope }\end{array}$ & $1 \mathrm{y}$ \\
\hline Aly et al, $2010^{20}$ & Not described & N/A & $\begin{array}{l}\text { Dysphagia was assessed using a } \\
\text { previously validated scoring } \\
\text { system based on a 9-item graded } \\
\text { food scale with no dysphagia } \\
\text { scoring } 0 \text { and a maximum score of } \\
457 \text { as well as a } 0-10 \text { analog scale. } \\
\text { - Any form of anastomotic } \\
\text { narrowing requiring endoscopic } \\
\text { dilatation or operative revision }\end{array}$ & $1 \mathrm{y}$ \\
\hline Ma et al, $2010^{27}$ & N/A & N/A & $\begin{array}{l}\text { - Radiographic anastomotic } \\
\text { diameter }<0.8 \mathrm{~cm}\end{array}$ & $3 \mathrm{mo}$ \\
\hline Nederlof et al, $2011^{12}$ & $\begin{array}{l}\text { - Drain output of intestinal fluids } \\
\text { - Any radiographic } \\
\text { - Any endoscopic evidence }\end{array}$ & $\begin{array}{l}\text { 6th postoperative day swallow } \\
\text { study and } 7 \text { th postoperative } \\
\text { day endoscopy }\end{array}$ & $\begin{array}{l}\text { - Inability to proceed into the gastric } \\
\text { tube with a } 9 \mathrm{~mm} \text { endoscope }\end{array}$ & $12 \mathrm{mo}$ \\
\hline
\end{tabular}


TABLE 2. Continued

\begin{tabular}{|c|c|c|c|c|}
\hline Study & Anastomotic leak diagnosis & $\begin{array}{c}\text { Routine postoperative } \\
\text { anastomotic assessment }\end{array}$ & Stricture diagnosis & $\begin{array}{c}\text { Last } \\
\text { follow-up }\end{array}$ \\
\hline Liu et al, $2011^{21}$ & N/A & N/A & N/A & N/A \\
\hline Saluja et al, $2012^{13}$ & $\begin{array}{l}\text { - Drain output of intestinal fluids } \\
\text { - Any radiographic evidence }\end{array}$ & $\begin{array}{l}\text { 7th postoperative day swallow } \\
\text { study }\end{array}$ & Not described & $3 \mathrm{y}$ \\
\hline Cayi et al, $2012^{14}$ & N/A & N/A & N/A & N/A \\
\hline Wang et al, $2013^{22}$ & N/A & N/A & $\begin{array}{l}\text { - Radiographic anastomotic } \\
\text { diameter }<0.8 \mathrm{~cm}\end{array}$ & $\begin{array}{c}3 \text { mo after } \\
\text { surgery }\end{array}$ \\
\hline Hayata et al, $2017^{16}$ & $\begin{array}{l}\text { - Drain output of intestinal fluids } \\
\text { - Any radiographic evidence } \\
\text { - Any endoscopic evidence }\end{array}$ & $\begin{array}{l}\text { 7th postoperative day swallow } \\
\text { study, endoscopy and CT }\end{array}$ & $\begin{array}{l}\text { - Inability to proceed into the gastric } \\
\text { tube with a } 9 \mathrm{~mm} \text { endoscope }\end{array}$ & $12 \mathrm{mo}$ \\
\hline
\end{tabular}

N/A, Not available; $C T$, computed tomography. ${ }^{*}$ Swallow esophagogram with either water-soluble or barium contrast.

(OR, 0.95; 95\% CI, 0.51-177). ${ }^{9-16}$ The full forest plot of these studies can be appreciated in Figure E2.

\section{DISCUSSION}

This systematic review and meta-analysis of 17 randomized controlled trials (RCTs) with 2230 patients shows that hand-sewn anastomosis is associated with a higher rate of $\mathrm{AL}$ and anastomotic stricture, but no difference in 30-day mortality as illustrated in Figure 7 and summarized in Figure 8 and Video 1.

The strengths of this study are inclusion of only RCTs and the associated extensive literature search, resulting in

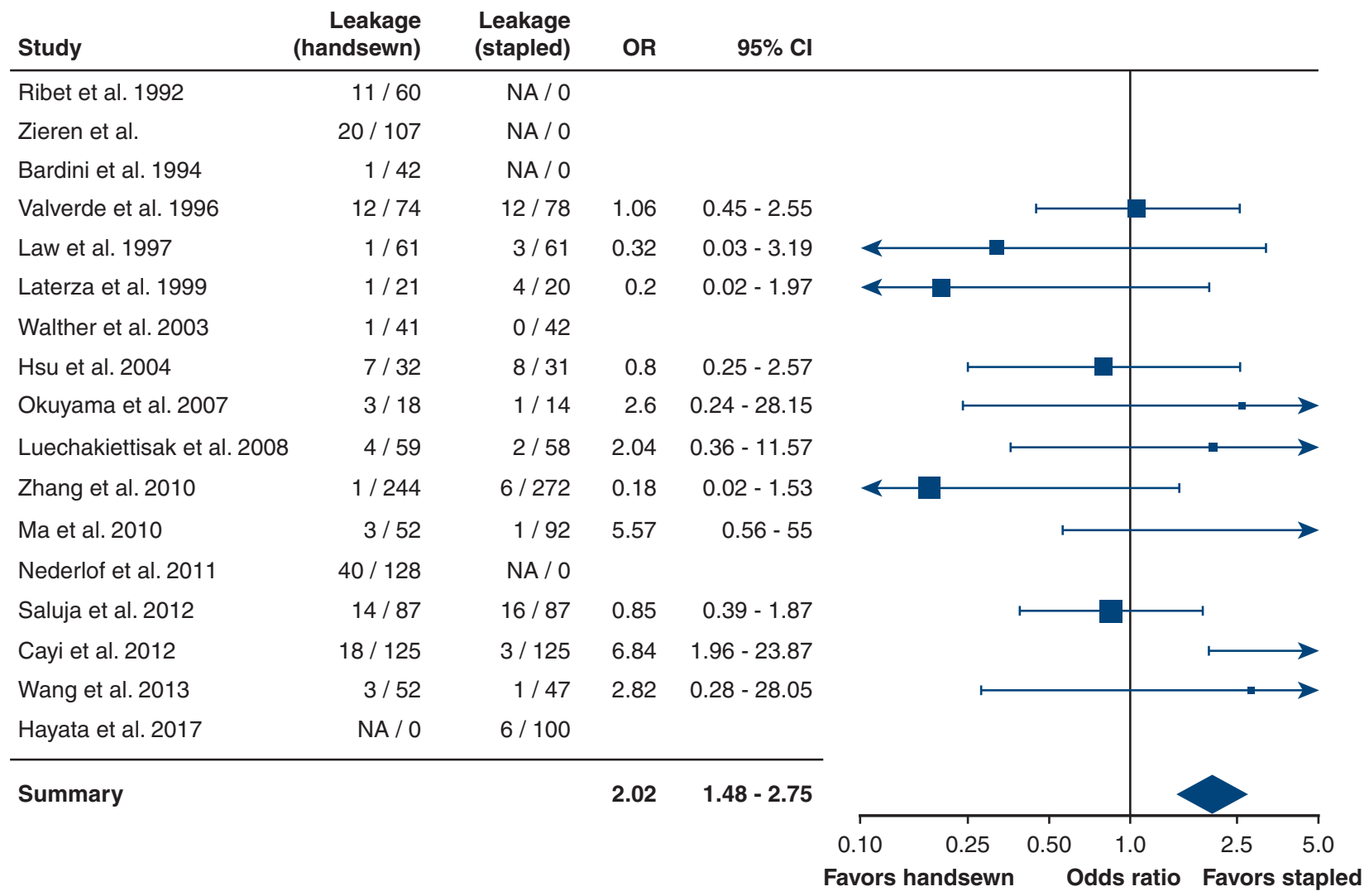

FIGURE 2. Forest plot for anastomotic leak comparing hand-sewn anastomosis to stapled anastomosis. OR, Odds ratio; $C I$, confidence interval; $N A$, not available. 


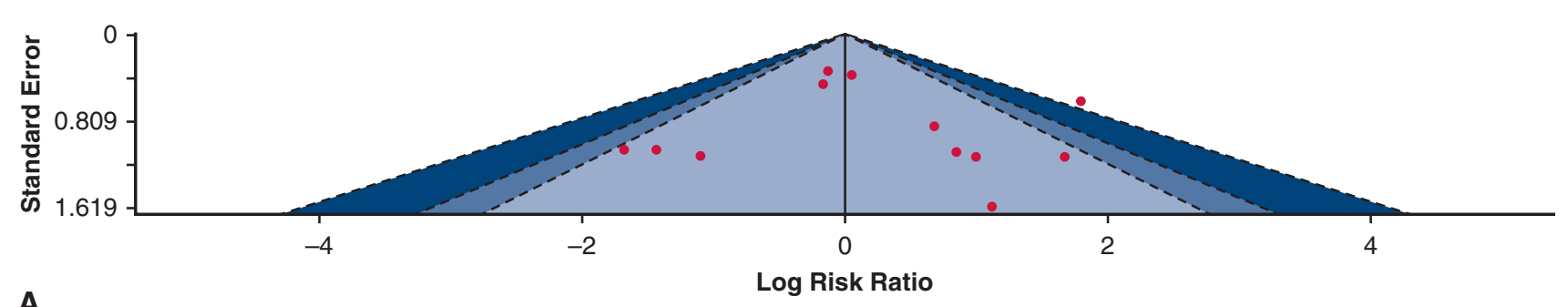

A

Log Risk Ratio

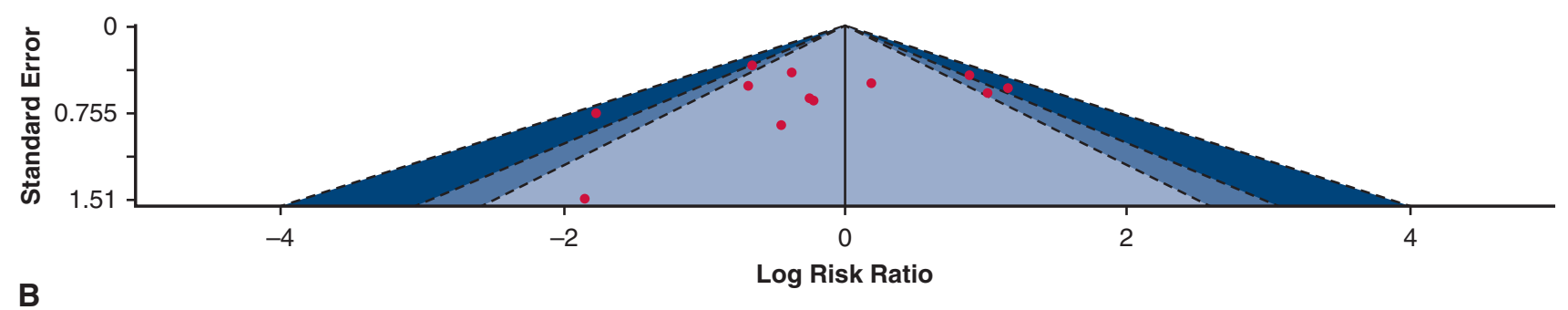

$\mathbf{B}$

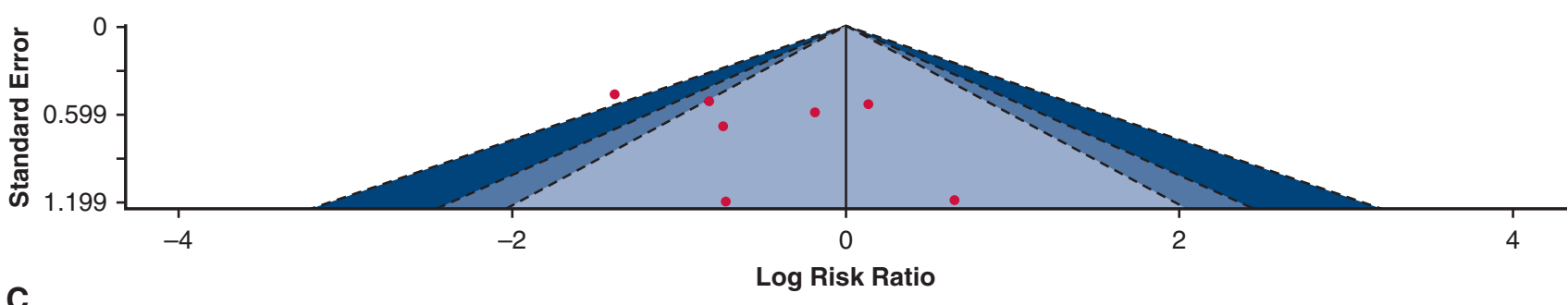

C

$$
\square 0.1<\mathrm{p}<1.0 \square 0.05<\mathrm{p}<0.1 \square 0.04<\mathrm{p}<0.05 \square 0<\mathrm{p} 0.04
$$

FIGURE 3. Funnel plots for (A) anastomotic leak, (B) anastomotic stricture, and (C) 30-day mortality.

a large number of applicants and minimal confounding by patient-related prognostic factors. Limitations include the heterogeneity of reported outcomes in the RCTs, especially in regard to mortality, which was either not reported, reported as in-hospital mortality, or 30-day mortality. To facilitate comparisons among groups, 30-day all-cause mortality was used. The studies included were performed over a 25 -year time span, which introduces more heterogeneity in the form of different neoadjuvant, perioperative oncologic therapies and overall postoperative treatment protocols. The difference of patient populations and disease characteristics between Asian and Western studies is a probable source of bias. Studies that used either only hand-sewn or stapled anastomoses were included in the meta-analysis, which might exaggerate different reporting criteria between the studies. Different surgical methods were employed (eg, single-layer or 2-layer anastomosis, circular [end-to-end anastomosis] or side-stapled anastomosis), and the effects of these variations in methods are hard to quantify. Moreover, the superiority between these specific techniques cannot be established from this study and should be further investigated in future studies/reports.
Studies included in this trial had mostly a moderate risk of bias, as shown with Cochrane Collaborations Risk of Bias Tool analysis because with surgical clinical studies, the masking of the intervention from the subjects and the care team is difficult or even impossible. No studies tried to analyze or to account for this bias. Very limited description of the randomization process was available and use of blinded investigators in the analysis of data was rare.

The sensitivity analyses show some variance in results compared with the original meta-analysis. The inclusion of the single group-studies into the quantitative analysis favored the stapled anastomosis groups because without these groups in the analysis, intergroup differences in $\mathrm{AL}$ rate become nonsignificant and 30-day mortality then favors hand-sewn anastomoses. When interpreting these results, one must note that some of the included RCTs compared intrathoracic stapled anastomoses to hand-sewn cervical anastomoses. ${ }^{23,25,26}$ Intrathoracic anastomosis requires intrathoracic entry, and because many of these studies were done before the widespread use of minimally invasive techniques, this meant performing a thoracotomy, which has been shown to negatively influence outcomes 


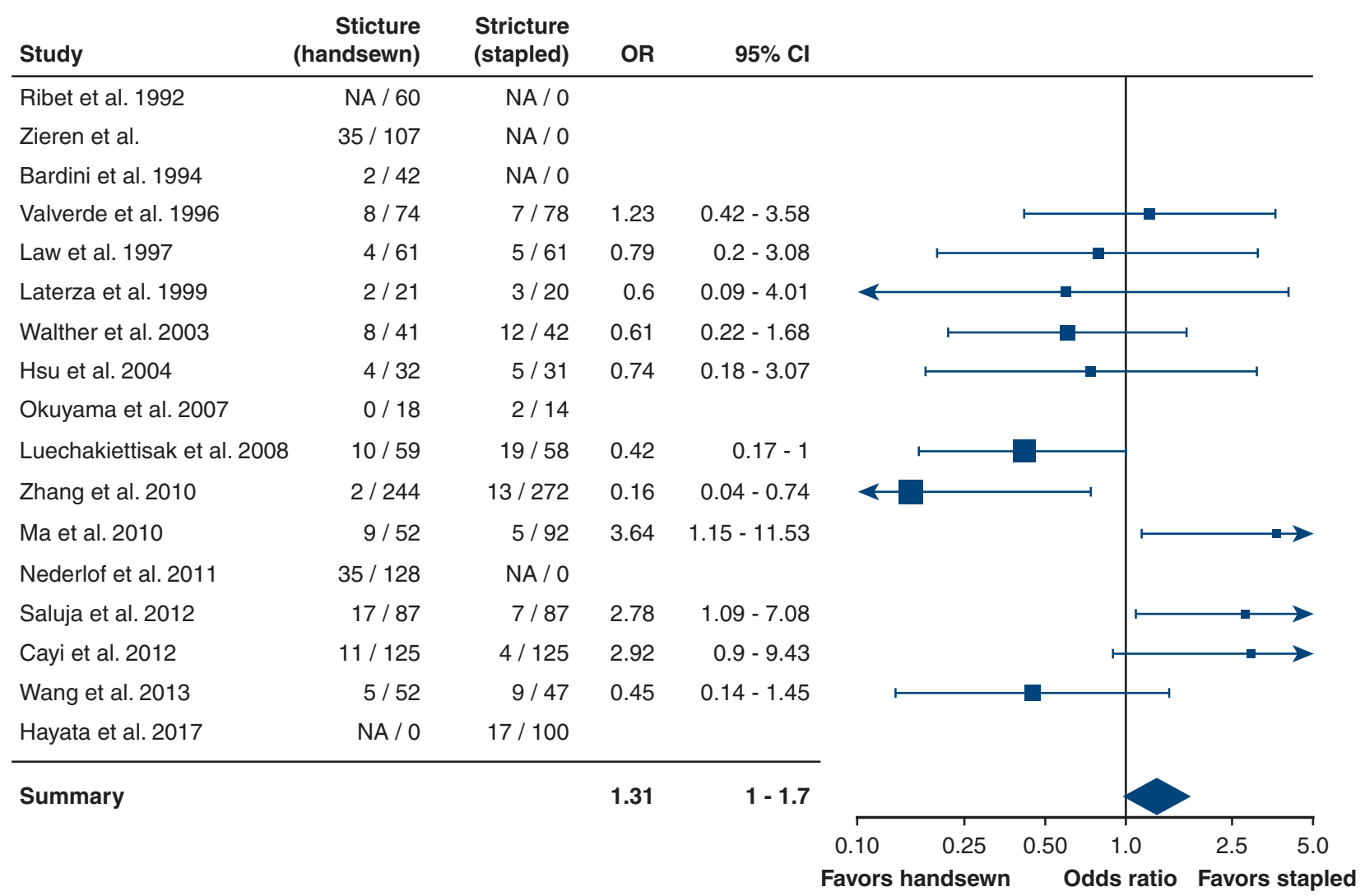

FIGURE 4. Forest plot for anastomotic stricture comparing hand-sewn anastomosis to stapled anastomosis. $O R$, Odds ratio; $C I$, confidence interval; $N A$, not available.

compared with minimally invasive techniques. ${ }^{2}$ In fact, among the studies included, only 1 study applied minimally invasive techniques. ${ }^{16}$ In contrast, the majority of cervical anastomosis were done via a transhiatal approach (390 transhiatal esophagectomies vs 287 McKeown esophagectomies), which spares the patient thoracotomy-related morbidity, but at the expense of thoracic lymph node yield, possibly sacrificing long-term survival. ${ }^{28,29}$ This might bias the results of the 30-day mortality to favor the hand-sewn anastomosis group because the comparison is between operations and surgical risk with thoracotomy and transhiatal esophagectomies. When observing only thoracic anastomoses, no difference between the anastomotic techniques could be established in any of the outcomes. The results of the sensitivity analyses of thoracic-only anastomoses are subject to possible random error because only 4 studies used exclusively thoracic anastomoses. The same can be said of the OS results of cervical anastomoses because only 2 studies of this group reported 30-day mortality. Exclusion of studies conducted before the turn of the millennium did not change the summary statistics results, speaking against any significant confounding effect of studies published earlier.
The reported rates of AL between the studies varied between $1.2 \%$ and $31.3 \%$. Definitions for AL were heterogeneous, or not elucidated in the article, which most likely explains the variability of the incidence and produces more heterogeneity to this analysis. Explanation for high degree of difference for the difference in stricture rates between the techniques when used in the thorax or in the cervical region is not known to the authors. It may be possible that circular stapling and side-to-side stapling provide different rates of stricture and/or AL, which may confound the findings of this meta-analysis. Unfortunately, due to the low amount of studies reporting the use of side-to-side stapling, such a separate analysis between the techniques is not feasible. ${ }^{13,16,27}$

Studies did not report whether their institutions, or the surgeons, had a preferred anastomotic technique. If there is a preferred technique that is in routine use at the institution, the alternative anastomotic technique used in the study might be subject to higher rate of complication and/or observation bias.

Although this meta-analysis includes a fair number of randomized controlled studies $(\mathrm{n}=19)$ and patients $(n=2308)$ there is clearly need for better quality data, if 


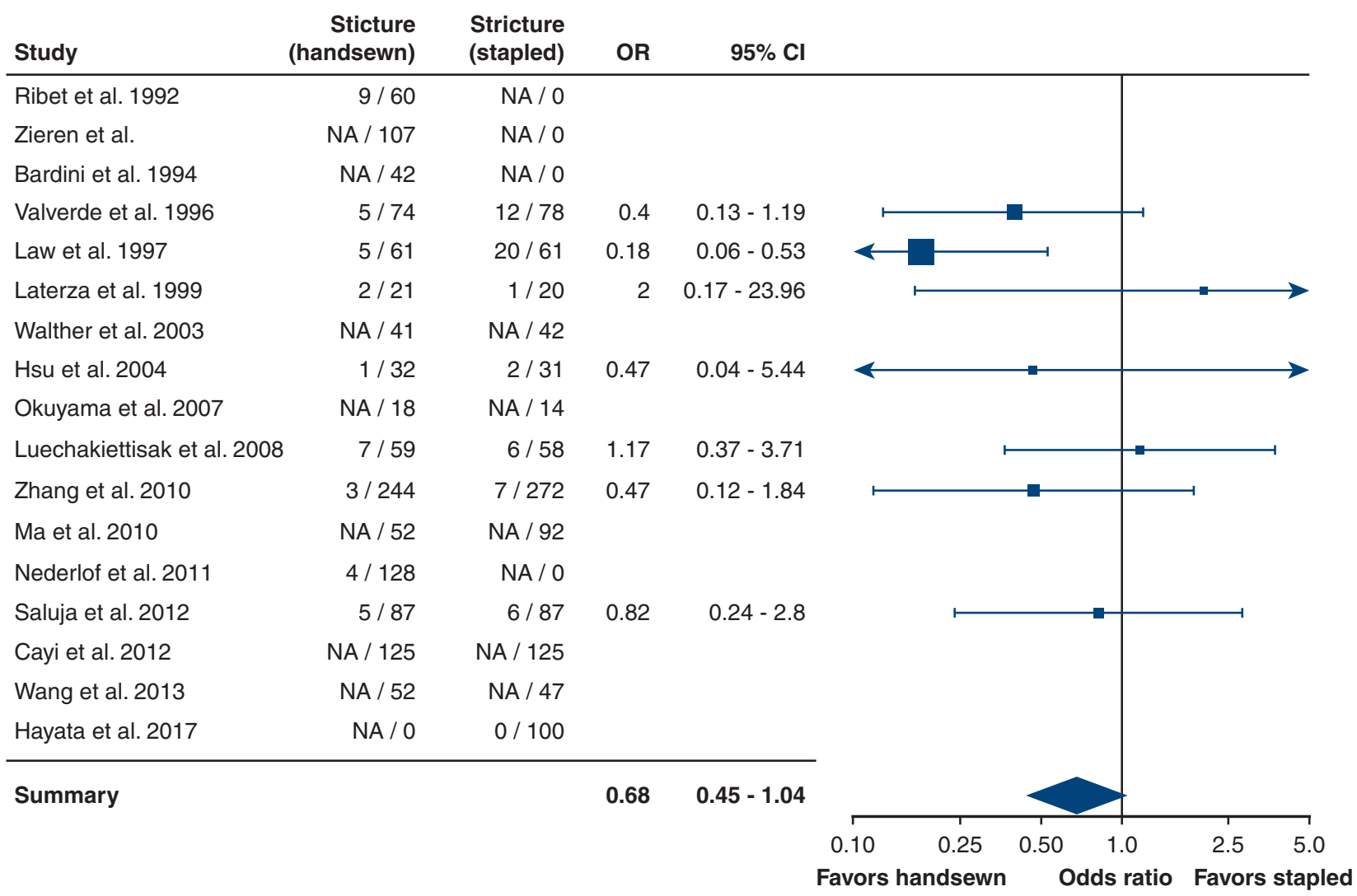

FIGURE 5. Forest plot for 30-day mortality comparing hand-sewn anastomosis to stapled anastomosis. OR, Odds ratio; $C I$, confidence interval; $N A$, not available.

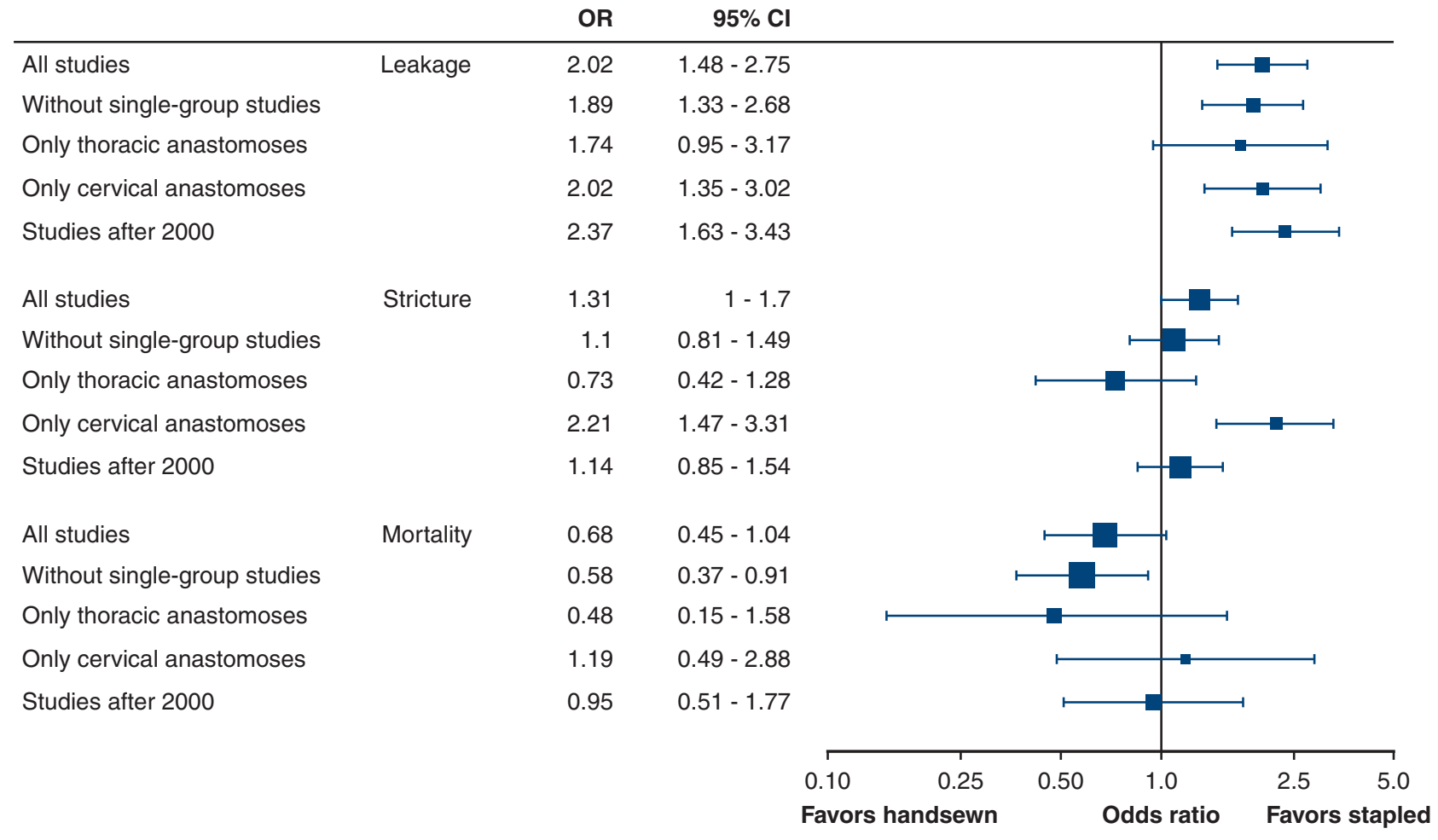

FIGURE 6. Sensitivity analyses of the anastomotic leak, anastomotic stricture, and 30-day mortality analyses. Sensitivity analyses included were analyses without single-group studies, thoracic anastomoses only, and cervical anastomoses only. OR, Odds ratio; $C I$, confidence interval. 


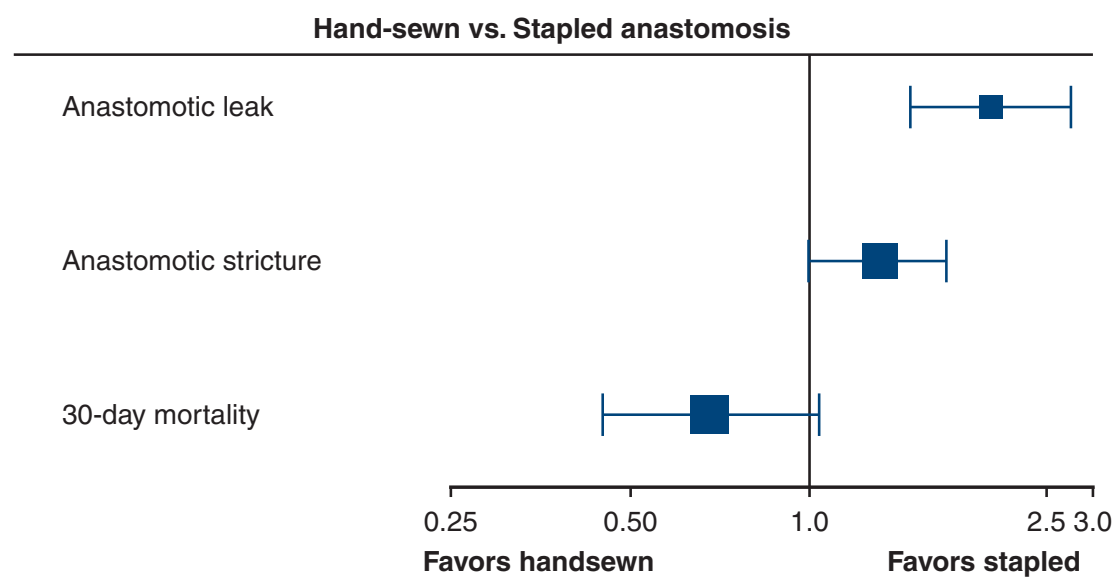

FIGURE 7. Forest plot showing the summary results for anastomotic leak, anastomotic stricture, and 30-day mortality.

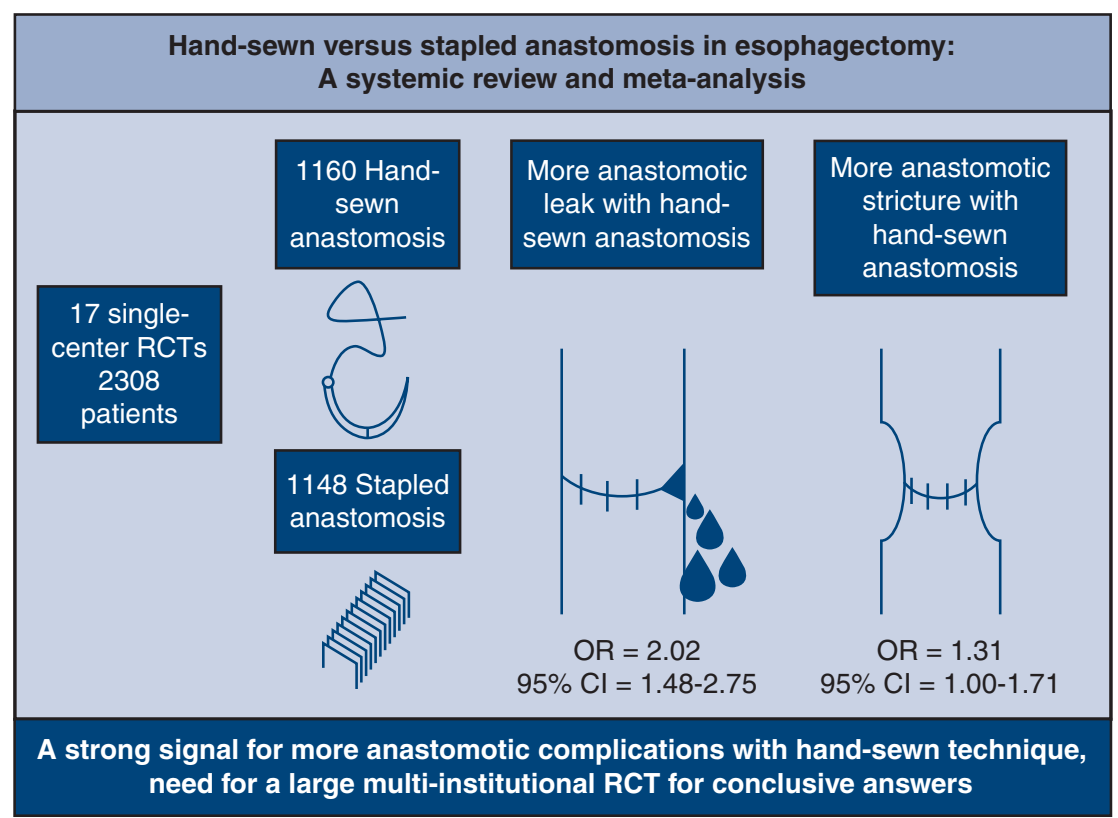

FIGURE 8. Graphical abstract summarizing the results of the study. From the left to right, Amount of randomized controlled trials (RCTs) and patients involved, number of patients in hand-sewn and stapled anastomosis groups, comparison of groups in anastomotic leak rates, and comparison of groups in anastomotic stricture rates. $O R$, Odds ratio; $C I$, confidence interval.

a definitive answer to the superiority of an anastomotic technique is to be proven. A large-scale multi-institutional RCT with clearly characterized and clinically meaningful outcomes could provide us with an answer, but for now the data can be interpreted in a multitude of ways. It could also be the case that the difference between the techniques is so subtle that a massive number of patients would be needed to ferret out a statistical difference between the anastomotic technique, in which case the difference would probably not be clinically significant.

\section{CONCLUSIONS}

This meta-analysis shows that RCTs of esophageal anastomotic techniques are heterogeneous with a risk of bias, and paucity of data in the minimally invasive setting. Our main finding is that there seems to be a signal favoring stapled anastomoses; however, these results show some discrepancy when subjected to sensitivity analyses and thus, no real recommendation of a preferred anastomotic technique can be made. We hope that this meta-analysis underscores the need for modern, well-performed RCTs. In 


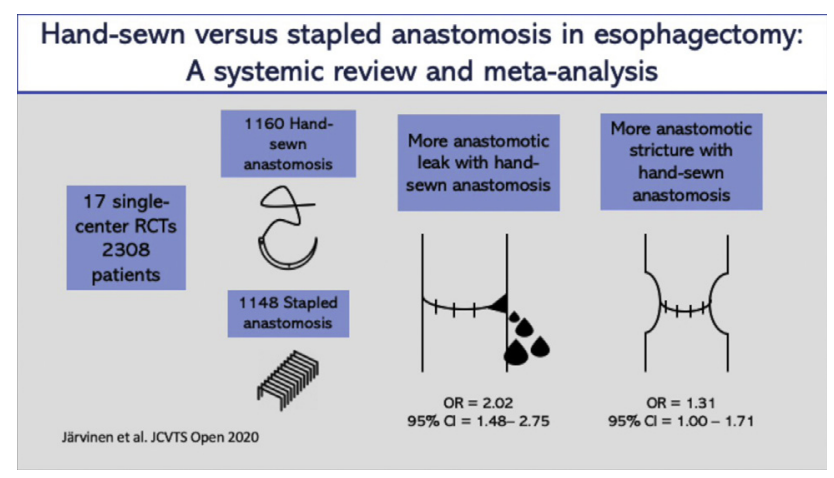

VIDEO 1. The corresponding author, Tommi Järvinen, summarizes the results of the meta-analysis. Video available at: https://www.jtcvs.org/article/ S2666-2736(21)00207-2/fulltext.

the end, the authors all agree that the most important factor in anastomotic technique is a well-vascularized anastomosis constructed without tension rather than a specific anastomotic technique.

\section{Conflict of Interest Statement}

The authors reported no conflicts of interest.

The Journal policy requires editors and reviewers to disclose conflicts of interest and to decline handling or reviewing manuscripts for which they may have a conflict of interest. The editors and reviewers of this article have no conflicts of interest.

\section{References}

1. Lagergren J, Smyth E, Cunningham D, Lagergren P. Oesophageal cancer. Lancet. 2017:390:2383-96

2. Gottlieb-Vedi E, Kauppila JH, Malietzis G, Nilsson M, Markar SR, Lagergren J. Long-term survival in esophageal cancer after minimally invasive compared to open esophagectomy a systematic review and meta-analysis. Ann Surg. 2019; 270:1005-17.

3. Biere SS, van Berge Henegouwen MI, Maas KW, Bonavina L, Rosman C, Garcia JR, et al. Minimally invasive versus open oesophagectomy for patients with oesophageal cancer: a multicentre, open-label, randomised controlled trial. Lancet. 2012;379:1887-92.

4. Maas KW, Cuesta MA, Henegouwen MIV, Roig J, Bonavina L, Rosman C, et al. Quality of life and late complications after minimally invasive compared to open esophagectomy: results of a randomized trial. World J Surg. 2015;39: 1986-93.

5. Biere SS, Maas KW, Cuesta MA, van der Peet DL. Cervical or thoracic anastomosis after esophagectomy for cancer: a systematic review and meta-analysis. Dig Surg. 2011;28:29-35.

6. Alanezi K, Urschel JD. Mortality secondary to esophageal anastomotic leak. Ann Thorac Cardiovasc Surg. 2004;10:71-5.

7. Moher D, Liberati A, Tetzlaff J, Altman DG, Prisma Group. Preferred reporting items for systematic reviews and meta-analyses: the PRISMA Statement. Ann Intern Med. 2009;151:264-W264.

8. Egger M, Davey Smith G, Schneider M, Minder C. Bias in meta-analysis detected by a simple, graphical test. BMJ. 1997;315:629-34.

9. Zieren HU, Muller JM, Pichlmaier H. Prospective randomized study of one-layer or 2-layer anastomosis following esophageal resection and cervical esophagogastrostomy. Br J Surg. 1993;80:608-11.

10. Bardini R, Bonavina L, Asolati M, Ruol A, Castoro C, Tiso E. Single-layered cervical esophageal anastomoses: a prospective study of two suturing techniques. Ann Thorac Surg. 1994;58:1087-9.
11. Laterza E, de’ Manzoni G, Veraldi GF, Guglielmi A, Tedesco P, Cordiano C. Manual compared with mechanical cervical oesophagogastric anastomosis: a randomised trial. Eur J Surg. 1999;165:1051-4.

12. Nederlof N, Tilanus HW, Tran TC, Hop WC, Wijnhoven BP, de Jonge J. End-toend versus end-to-side esophagogastrostomy after esophageal cancer resection: a prospective randomized study. Ann Surg. 2011;254:226-33.

13. Saluja SS, Ray S, Pal S, Sanyal S, Agrawal N, Dash NR, et al. Randomized trial comparing side-to-side stapled and hand-sewn esophagogastric anastomosis in neck. J Gastrointest Surg. 2012;16:1287-95.

14. Cayi R, Li M, Xiong G, Cai K, Wang W. Comparative analysis of mechanical and manual cervical esophagogastric anastomosis following esophagectomy for esophageal cancer. Nan Fang Yi Ke Da Хue Xue Bao. 2012;32:908-9.

15. Hsu HH, Chen JS, Huang PM, Lee JM, Lee YC. Comparison of manual and mechanical cervical esophagogastric anastomosis after esophageal resection for squamous cell carcinoma: a prospective randomized controlled trial. Eur J Cardiothorac Surg. 2004;25:1097-101.

16. Hayata K, Nakamori M, Nakamura M, Ojima T, Iwahashi M, Katsuda M, et al. Circular stapling versus triangulating stapling for the cervical esophagogastric anastomosis after esophagectomy in patients with thoracic esophageal cancer: a prospective, randomized, controlled trial. Surgery. 2017;162:131-8.

17. Law S, Fok M, Chu KM, Wong J. Comparison of hand-sewn and stapled esophagogastric anastomosis after esophageal resection for cancer: a prospective randomized controlled trial. Ann Surg. 1997;226:169-73.

18. Luechakiettisak P, Kasetsunthorn S. Comparison of hand-sewn and stapled in esophagogastric anastomosis after esophageal cancer resection: a prospective randomized study. J Med Assoc Thai. 2008;91:681-5.

19. Zhang YS, Gao BR, Wang HJ, Su YF, Yang YZ, Zhang JH, et al. Comparison of anastomotic leakage and stricture formation following layered and stapler oesophagogastric anastomosis for cancer: a prospective randomized controlled trial. $J$ Int Med Res. 2010;38:227-33.

20. Aly A, Jamieson GG, Watson DI, Devitt PG, Ackroyd R, Stoddard CJ. An antireflux anastomosis following esophagectomy: a randomized controlled trial. $J$ Gastrointest Surg. 2010;14:470-5.

21. Liu JF, Wang JD, Liu XB, Sun YH, Jiang T, Wang FS, et al. Antireflux anastomosis following resection of esophageal cancer. Zhonghua Wai Ke Za Zhi. 2011;49:61-5.

22. Wang WP, Gao Q, Wang KN, Shi H, Chen LQ. A prospective randomized controlled trial of semi-mechanical versus hand-sewn or circular stapled esophagogastrostomy for prevention of anastomotic stricture. World J Surg. 2013;37: 1043-50.

23. Ribet M, Debrueres B, Lecomte-Houcke M. Resection for advanced cancer of the thoracic esophagus: cervical or thoracic anastomosis? Late results of a prospective randomized study. J Thorac Cardiovasc Surg. 1992;103:784-9.

24. Valverde A, Hay JM, Fingerhut A, Elhadad A. Manual versus mechanical esophagogastric anastomosis after resection for carcinoma: a controlled trial. French Associations for Surgical Research. Surgery. 1996;120:476-83.

25. Walther B, Johansson J, Johnsson F, Von Holstein CS, Zilling T. Cervical or thoracic anastomosis after esophageal resection and gastric tube reconstruction: a prospective randomized trial comparing sutured neck anastomosis with stapled intrathoracic anastomosis. Ann Surg. 2003;238:803-12.

26. Okuyama M, Motoyama S, Suzuki H, Saito R, Maruyama K, Ogawa J. Hand-sewn cervical anastomosis versus stapled intrathoracic anastomosis after esophagectomy for middle or lower thoracic esophageal cancer: a prospective randomized controlled study. Surg Today. 2007;37:947-52.

27. Ma RD, Zhang WT, Xu QR, Chen LQ. Esophagogastrostomy by side-to-side anastomosis in prevention of anastomotic stricture: a randomized clinical trial. Zhonghua Wai Ke Za Zhi. 2010;48:577-81.

28. Peyre CG, Hagen JA, DeMeester SR, Altorki NK, Ancona E, Griffin SM, et al. The number of lymph nodes removed predicts survival in esophageal cancer: an international study on the impact of extent of surgical resection. Ann Surg. 2008;248:549-56.

29. Kutup A, Nentwich MF, Bollschweiler E, Bogoevski D, Izbicki JR, Holscher AH. What should be the gold standard for the surgical component in the treatment of locally advanced esophageal cancer transthoracic versus transhiatal esophagectomy. Ann Surg. 2014;260:1016-22.

Key Words: esophagectomy, anastomosis, meta-analysis, systematic review, esophageal surgery 


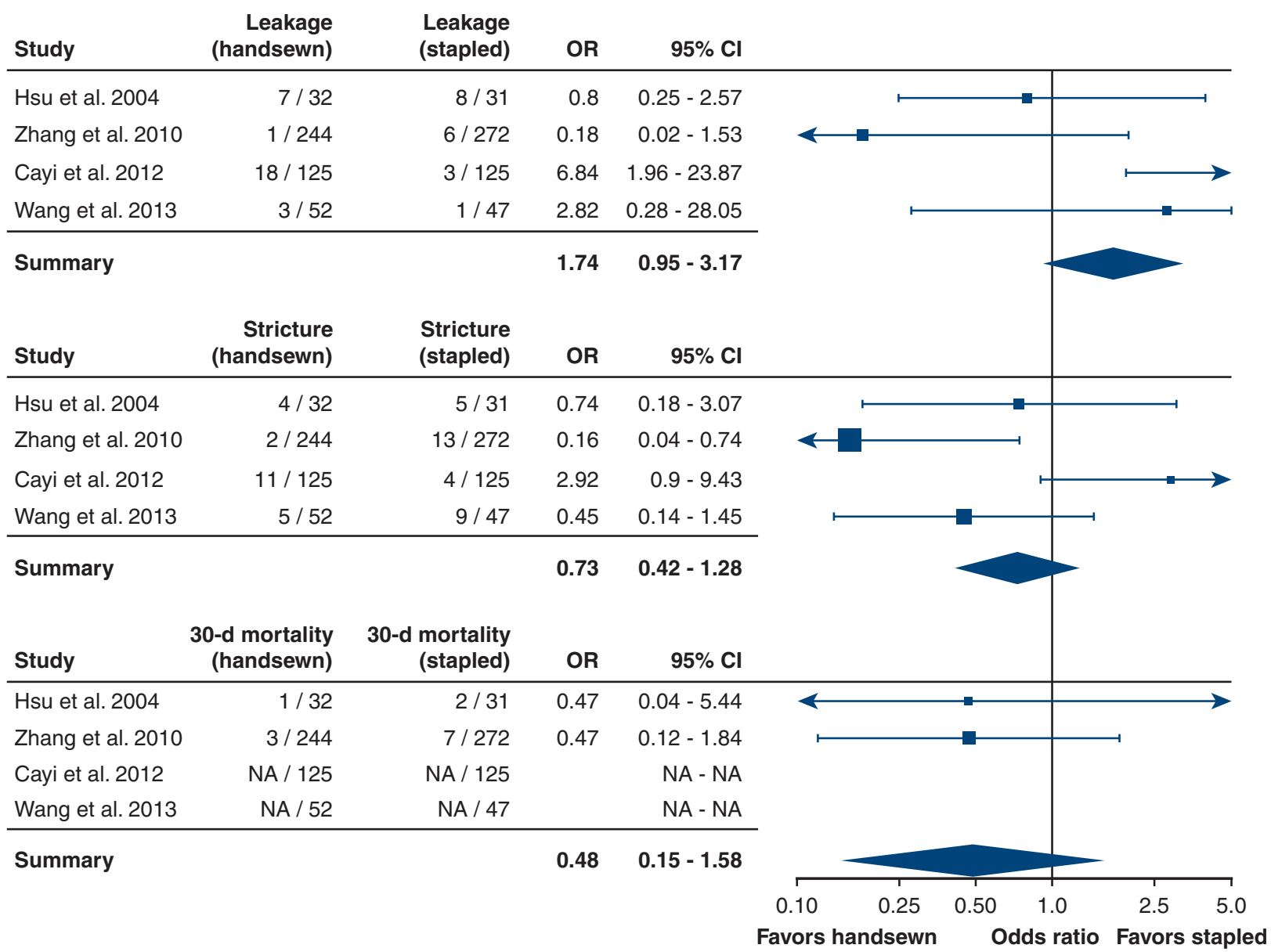

FIGURE E1. Forest plots of thoracic anastomosis-only studies. $O R$, Odds ratio; $C I$, confidence interval; $N A$, not available. 


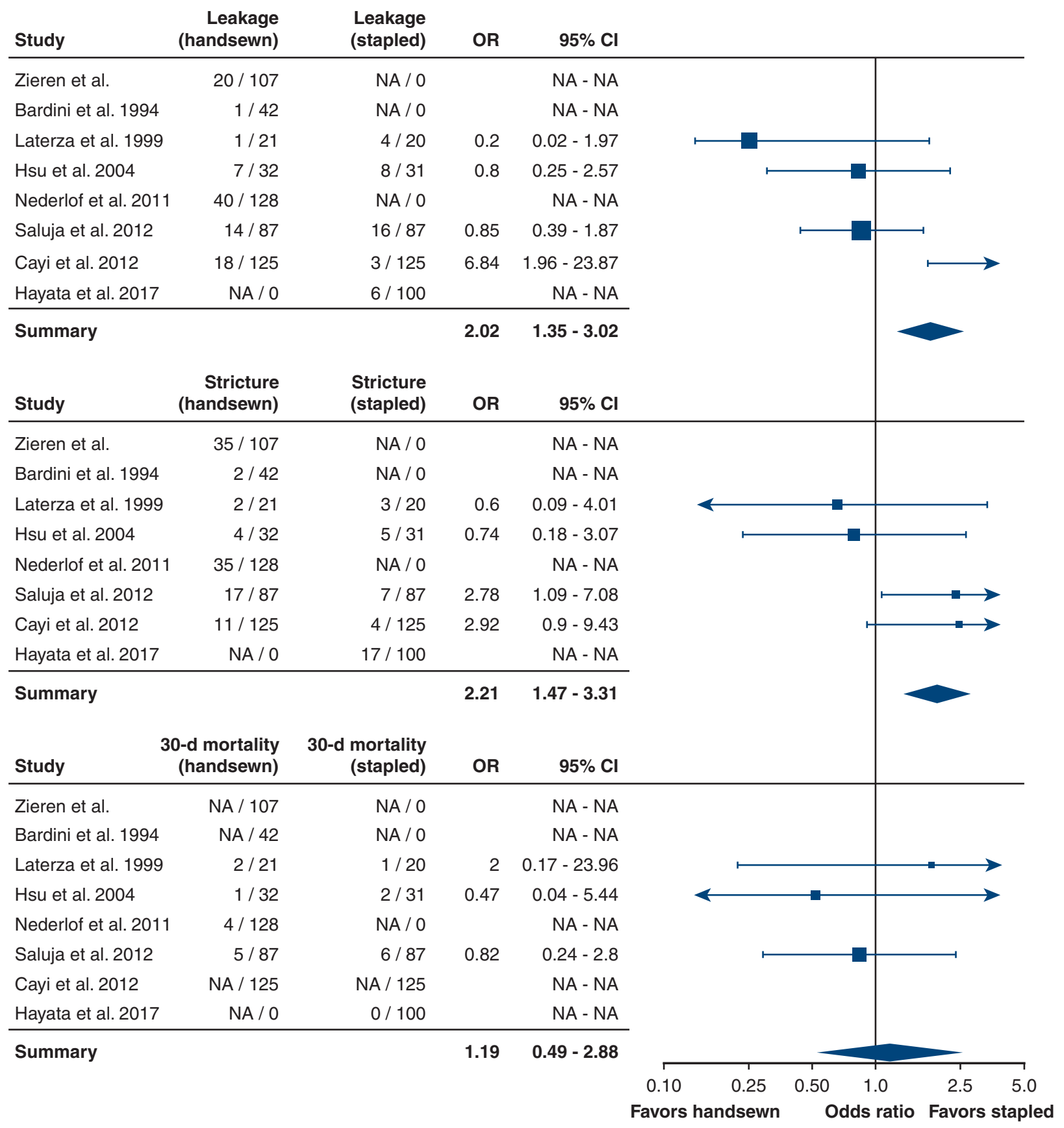

FIGURE E2. Forest plots of cervical anastomosis-only studies. $O R$, Odds ratio; $C I$, confidence interval; $N A$, not available. 
TABLE E1. Electronic search strategies

\begin{tabular}{|c|c|}
\hline Medline & Scopus \\
\hline 1. exp Esophageal neoplasms/ & 1. TITLE-ABS-KEY ( esoph*) \\
\hline $\begin{array}{l}\text { 2. ((esophag* or oesophag*) adj3 (cancer* or neoplas* or carcin* or } \\
\text { adenocarcin* or tumour* or tumor* or malig*)).ti,ab,kf. }\end{array}$ & 2. TITLE-ABS-KEY(cancer OR carc* OR malig* OR neoplas*) \\
\hline 3. or/1-2 & 3. 1 and 2 \\
\hline 4. exp Anastomosis, surgical/ & 4. TITLE-ABS-KEY ( anastomo*) \\
\hline 5. (anastomo*).ti,ab,kf & 5.3 and 4 \\
\hline 6. or/4-5 & 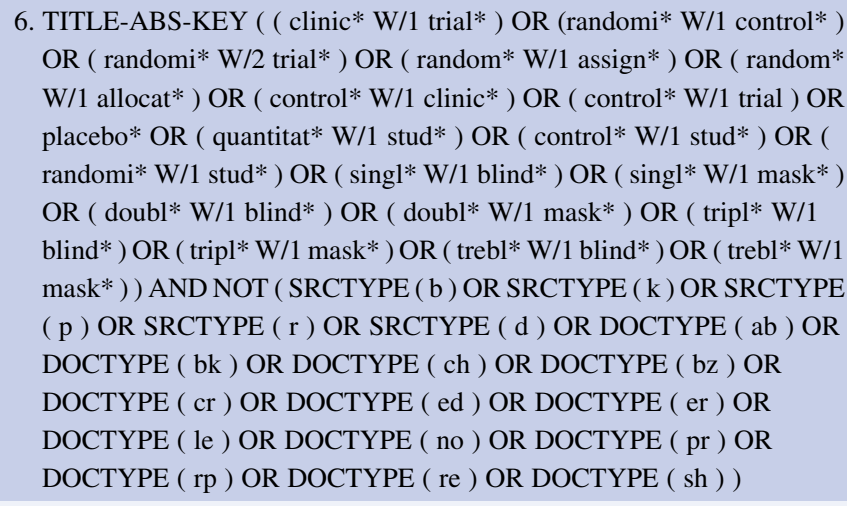 \\
\hline 7. 3 and 6 & 7. 5 and 6 \\
\hline OvidSp & CENTRAL \\
\hline 1. exp Esophageal neoplasms/ & $\begin{array}{l}\text { 1. ((esophag* or oesophag* or gastroesophag* or gastrooesophag*) near/3 } \\
\text { (cancer* or neoplas* or carcin* or adenocarcin* or tumour* or tumor* or } \\
\text { malig*)):ab,ti,kw }\end{array}$ \\
\hline $\begin{array}{l}\text { 2. ((esophag* or oesophag*) adj3 (cancer* or neoplas* or carcin* or } \\
\text { adenocarcin* or tumour* or tumor* or malig*)).ti,ab,kf. }\end{array}$ & 2. (anastom*):ab,ti,kw \\
\hline 3. or/1-2 & 3. \#1 and \#2 \\
\hline \multicolumn{2}{|l|}{ 4. exp Anastomosis, surgical/ } \\
\hline \multicolumn{2}{|l|}{ 5. (anastomo*).ti,ab,kf } \\
\hline \multicolumn{2}{|l|}{ 6. or/4-5 } \\
\hline 7. 3 and 6 & \\
\hline
\end{tabular}


TABLE E2. Risk of bias using Cochrane Collaborations Risk of Bias Tool

\begin{tabular}{|c|c|c|c|c|c|c|c|}
\hline $\begin{array}{c}\text { Study } \\
\text { ID }\end{array}$ & Author and year & $\begin{array}{c}\text { Randomization } \\
\text { process }\end{array}$ & $\begin{array}{c}\text { Deviations } \\
\text { from intended } \\
\text { interventions }\end{array}$ & $\begin{array}{c}\text { Missing } \\
\text { outcome } \\
\text { data }\end{array}$ & $\begin{array}{l}\text { Measurement } \\
\text { of the outcome }\end{array}$ & $\begin{array}{l}\text { Selection of the } \\
\text { reported result }\end{array}$ & Overall bias \\
\hline 1 & Hsu et al, $2004^{15}$ & Some concerns & Some concerns & Low & Some concerns & Low & Some concerns \\
\hline 2 & Law et al, $1997^{17}$ & Some concerns & Some concerns & Low & Low & Low & Some concerns \\
\hline 3 & Okuyama et al, $2007^{26}$ & Some concerns & High & Low & Low & Low & High \\
\hline 4 & $\begin{array}{l}\text { Luechakiettisak et al, } \\
2008^{18}\end{array}$ & Some concerns & Some concerns & $\begin{array}{l}\text { Some } \\
\text { concerns }\end{array}$ & Low & Some concerns & Some concerns \\
\hline 5 & Wang et al, $2013^{22}$ & High & Some concerns & Low & Low & Low & High \\
\hline 6 & Zhang et al, $2010^{19}$ & Some concerns & Some concerns & Low & Low & Low & Some concerns \\
\hline 7 & Zieren et al, $1993^{9}$ & Low & Some concerns & Low & Some concerns & Some concerns & Some concerns \\
\hline 9 & Saluja et al, $2012^{13}$ & Some concerns & Some concerns & Low & Some concerns & Some concerns & Some concerns \\
\hline 10 & Walther et al, $2003^{25}$ & Some concerns & Some concerns & Low & Some concerns & Some concerns & Some concerns \\
\hline 11 & Nederlof et al, $2011^{12}$ & Low & Some concerns & Low & Low & Low & Low \\
\hline 12 & Ma et al, $2010^{27}$ & Some concerns & Some concerns & Low & Some concerns & Some concerns & Some concerns \\
\hline 13 & Ribet et al, $1992^{23}$ & High & High & Low & Some concerns & Some concerns & High \\
\hline 14 & Valverde et al, $1996^{24}$ & Low & Some concerns & Low & Low & Some concerns & Some concerns \\
\hline 17 & Bardini et al, $1994^{10}$ & Some concerns & Some concerns & Low & Low & Some concerns & Some concerns \\
\hline 18 & Laterza et al, $1999^{11}$ & Low & Some concerns & Low & Low & Some concerns & Some concerns \\
\hline 20 & Hayata et al, $2017^{16}$ & Low & Some concerns & Low & Low & Low & Low \\
\hline 21 & Cayi et al, $2012^{14}$ & Some concerns & Some concerns & Low & Low & Some concerns & Some concerns \\
\hline
\end{tabular}

